\title{
Pierre-Nicolas CHANTREAU, Dictionnaire national et anecdotique (1790)
}

Présenté et annoté par Agnès STEUCKARDT, Limoges, Lambert-Lucas, Collection La Lexicothèque, 2008, 223 p., ISBN 978-2-915806-78-6, $30 €$

\section{Raymonde Monnier}

\section{OpenEdition}

\section{Journals}

\section{Édition électronique}

URL : https://journals.openedition.org/ahrf/11579

DOI : 10.4000/ahrf.11579

ISSN : 1952-403X

Éditeur:

Armand Colin, Société des études robespierristes

\section{Édition imprimée}

Date de publication : 1 octobre 2009

Pagination : 191-193

ISBN : 978-2-200-92560-4

ISSN : 0003-4436

\section{Référence électronique}

Raymonde Monnier, «Pierre-Nicolas chantreau, Dictionnaire national et anecdotique (1790) ", Annales historiques de la Révolution française [En ligne], 358 | octobre-décembre 2009, mis en ligne le 29 juillet 2010, consulté le 24 avril 2022. URL : http://journals.openedition.org/ahrf/11579 ; DOI : https:// doi.org/10.4000/ahrf.11579

Ce document a été généré automatiquement le 24 avril 2022.

Tous droits réservés 


\section{Pierre-Nicolas CHANTREAU, Dictionnaire national et anecdotique (1790)}

Présenté et annoté par Agnès STEUCKARDT, Limoges, Lambert-Lucas, Collection La Lexicothèque, 2008, 223 p., ISBN 978-2-915806-78-6, $30 €$

\section{Raymonde Monnier}

\section{RÉFÉRENCE}

Pierre-Nicolas CHANTREAU, Dictionnaire national et anecdotique (1790), présenté et annoté par Agnès STEUCKARDT, Limoges, Lambert-Lucas, Collection La Lexicothèque, 2008, 223 p., ISBN 978-2-915806-78-6, $30 €$

On sait que les dictionnaires se sont multipliés pendant la période révolutionnaire pour enregistrer les changements de la langue, mais les linguistes ont généralement évité de se prononcer dans un débat longtemps orienté par des points de vue politiques. Agnès Steuckardt, connue des historiens de la Révolution pour ses études sur le lexique de la période et notamment sur L'Ami du Peuple de Marat, nous propose de juger sur pièces avec l'édition critique du premier dictionnaire de la période publié par un grammairien patriote qui a vécu l'événement et qui met le changement au cœur de son propos. Une introduction substantielle présente le Dictionnaire national et anecdotique, qui paraît en février ou mars 1790, dans le parcours social et intellectuel de son auteur et dans le contexte des dictionnaires de l'époque. Vient ensuite l'analyse des deux faces de l'ouvrage, son côté lexicographique et son côté discursif, ou plutôt satirique. PierreNicolas Chantreau (1741-1808), qui se dit ancien élève de Beauzée, a été avant la Révolution maître de langue à l'école militaire d'Avila, expérience qui l'amène à publier une grammaire qui restera en Espagne au XIX ${ }^{e}$ siècle la grammaire de référence pour l'enseignement du français. De retour à Paris dans les années 1780, il est vivement frappé de voir en 1789 la langue «s'enrichir chaque jour d'une foule de mots qui 
caractérisent un peuple libre ». C'est mû par le désir d'être utile à la nation qu'il entreprend, en homme de cabinet, les recherches pour son dictionnaire. Il fréquente les assemblées de district dont il décrit plaisamment les usages et suit l'actualité en lisant assidûment la presse; une notice de 24 pages donne des détails sur les différents journaux avant et depuis la Révolution. Écrit «pour servir à l'intelligence des mots dont notre langue s'est enrichie depuis la révolution » et « contenant les mots qui vont cesser d'être en usage, et qu'il est nécessaire d'insérer dans nos archives ", l'ouvrage rend compte du changement de manière piquante et originale. Il témoigne du patriotisme de son auteur, mais la satire n'épargne personne et si sa manière de jouer avec les mots suscite l'enthousiasme de Desmoulins, d'autres ont dû la trouver moins plaisante. Le citoyen Chantreau exploite ensuite un temps la veine du récit de voyage et fin 1793 quitte la capitale « pour un climat qui convint mieux à sa santé » en Gascogne, où il publie aux frais du département du Gers une feuille qui «sonne le tocsin » de la raison contre le christianisme. Après un essai sur les livres élémentaires, il devient professeur d'histoire à l'école centrale du Gers lors de sa création, puis à l'école militaire créée par Bonaparte à Fontainebleau; il publie alors essentiellement des ouvrages didactiques d'histoire.

2 Dans les dictionnaires alphabétiques de mots, qu'ils se prononcent sur les usages linguistiques et/ou sur les questions de société, le genre qui domine à la veille de 1789 est celui de la satire, illustré notamment par Rivarol. Les premiers dictionnaires de la Révolution sont néologiques; en poussant le genre vers la polémique politique, ils répondent à trois ressorts principaux : le plus fertile jusqu'en 1792 est celui de la réaction aristocratique aux nouveaux usages et à l'abus des mots. D'autres sont des dictionnaires néologiques raisonnés ou techniques dont le seul but est d'informer sur les termes nouveaux. Le Dictionnaire national et anecdotique, qui paraît comme les autres sans nom d'auteur, est le premier a afficher ses opinions révolutionnaires; c'est la première expertise alphabétique de quelque ampleur, entre linguistique et satire, des mots de la Révolution. Chantreau en prend le sens dans la variabilité des usages qu'il ordonne en fonction de leur situation historique: les usages du "nouveau régime» rendent ceux de l'« ancien régime » obsolètes. Ainsi « liberté »: «Dans l'ancien régime ce mot ne signifioit rien de ce qu'il signifie aujourd'hui qu'il est devenu le cri de la nation; il désigne ce droit précieux que nature accorde à tous les hommes [...] ». Une liste donne en appendice 185 mots ou expressions "qui vont cesser d'être en usage ", principalement les mots attachés à la société d'Ancien Régime dans le domaine politique ou religieux, ou plus caractéristique ceux de l'inégalité sociale (préséance, prérogative, privilège, protecteur ...). La plupart des mots listés n'ont en réalité pas disparu des dictionnaires mais se réfèrent à un passé révolu; ce qui n'est pas le cas pour ceux qui nomment une relation inégalitaire.

3 La nomenclature du Dictionnaire national et anecdotique prend en compte les innovations et le fonctionnement discursif de l'évolution sémantique : à côté des néolo-gismes formels, les mots jusque là peu employés et désormais entrés dans le discours quotidien (citoyen, district...), et des mots dont la Révolution a modifié le sens lexical (abus, nation...). Chantreau est conscient de la dette du lexique politique français à l'anglais ou au vocabulaire de la Rome antique, comme le mot " patrie » dont on n'avait l'idée qu'en sortant du collège et qui n'était ensuite " qu'un vain son » : « Mais aujourd'hui que nos courtisans sont épars [...] il y aura une patrie ; elle sera tout pour nous, nous serons tout pour elle ». Il se félicite d'être le premier à enregistrer le mot anglais « club » et fait à 
son sujet une remarque sur le mode plaisant : l'autre sens anglais étant coup de bâton, "si nous l'adoptons jamais dans ce sens, alors il ne pourra plus recevoir l'épithete de patriotique ». Il s'amuse à l'article «Anglois » du bon effet que doit faire sur ceux-ci «dans leurs objets d'importation en France» les mots constitutionnels; d'autres entrées suscitent une pointe d'impatience comme «loi martiale » qu'il n'aime guère : «Elle nous vient encore d'Angleterre!». Peu importe d'ailleurs l'origine d'un mot, ce qui compte est de l'illustrer au mieux : le mot « constitution " « étoit étranger avant la révolution ; l'assemblée nationale travaille à le rendre françois. La constitution formera le corps de loi qui convient à un peuple libre [...]». L'actualité fait émerger des acceptions inattendues (lanterne); des représentations nouvelles sont associées à certains termes au moment où les événements les mettent en vogue dans « les papiers publics» (cahiers, brigands, vaisselle d'argent). Dans les articles le goût du trait sociolinguistique ou l'émotion du rédacteur l'emportent sur la norme usuelle des définitions. Est-il même utile en 1790 de définir révolution : «Je n'ai pas besoin d'expliquer le mot, je n'ai que des vœux à faire pour la chose ». Une approche de son usage discursif est donné plus loin à l'article insurrection: "Ce qu'il y a de certain, c'est que quand l'insurrection est partielle et les insurgens au lieu de pendre sont pendus, il faut se servir pour parler exactement du mot de soulèvement. Dans le cas contraire et pour éviter toute équivoque, on emploie le mot révolution ».

4 La préoccupation majeure du rédacteur est l'usage pour pointer les changements, les mots à la mode et les variations diachroniques, selon le parti dont on est (incendiaire, libelle). La morphologie est prétexte à la satire contre l'Ancien Régime, ainsi s'agissant de «doléances »: «Il n'a point de singulier, et pour cause... »; la remarque piquante s'applique aussi au nouveau à propos du composé « bureaucratie »: « Observez qu'il est engendré par l'aristocratie ; jugez du monstre! ». Chantreau, « élève de feu M. Beauzée, Académicien, mort de l'Académie Française ", n'entend pas quant à lui périr de même et prévient la maladie par un discours préventif. Il conteste aux aristocrates le monopole de la satire en revendiquant la part de la "gaieté françoise» dans l'institution de la langue nationale. Pour mettre les rieurs de son côté, il persifle les inconséquences de l'Ancien Régime, tout en s'amusant des ridicules du nouveau dans un esprit d'autodérision. Son Dictionnaire national et anecdotique prend place dans la bataille du rire que se livrent en 1789 et 1790 partisans et adversaires de la Révolution et s'inscrit dans la série de textes qui placent la question de la langue au cœur du débat politique. Celui-ci est soigneusement annoté par A. Steuckardt, tant du point de vue historique que linguistique ; il est illustré et complété par une bibliographie des sources et travaux utilisés et par la liste des publications de son auteur. Laissons aux linguistes le soin de décider si la Révolution a changé ou non le français. Les historiens liront avec plaisir et intérêt ce discours idéologique et enjoué sur la langue de la Révolution : «Le choc des opinions amene souvent quelques aménités oratoires qui font perdre la gravité aux tribunes » (Opinant). «Chose incroyable, au milieu de ce fracas, un simple instrument rural que le pacifique bélier porte au cou et agite en marchant, une sonnette rétablit le calme ! [...] et tout finit comme l'a dit ce drôle de Figaro ». 